\title{
Downregulation of GPSM2 is associated with primary resistance to paclitaxel in breast cancer
}

\author{
ZHE ZHANG $^{1 *}$, ZHI LI $^{2 *}$, MINGMING DENG ${ }^{3,4^{*}}$, BOFANG LIU $^{5}$, XING XIN $^{6}$, \\ ZHENKUN ZHAO $^{1}$, YE ZHANG ${ }^{7}$ and QINGJIE $\mathrm{LV}^{1}$ \\ ${ }^{1}$ Department of Pathology, Shengjing Hospital of China Medical University; ${ }^{2}$ Department of Medical Oncology, \\ The First Hospital of China Medical University, Shenyang, Liaoning 110001; ${ }^{3}$ Department of Pulmonary and \\ Critical Care Medicine, Center of Respiratory Medicine, China-Japan Friendship Hospital; \\ ${ }^{4}$ The Graduate School of Peking Union Medical College, Chinese Academy of Medical Science and \\ Peking Union Medical College, Beijing 100029; ${ }^{5}$ Department of Medical Oncology, Sir Run Run Shaw Hospital, \\ College of Medicine, Zhejiang University, Hangzhou, Zhejiang 310016; ${ }^{6}$ Department of Medical Oncology, \\ The Fourth People's Hospital of Shenyang; ${ }^{7}$ The First Laboratory of The Cancer Institute, \\ The First Hospital of China Medical University, Shenyang, Liaoning 110001, P.R. China
}

Received August 14, 2019; Accepted December 18, 2019

DOI: $10.3892 /$ or.2020.7471

\begin{abstract}
Paclitaxel is one of the most effective chemotherapy drugs for breast cancer worldwide but $20-30 \%$ patients show primary resistance to the drug. Screening and identification of markers that facilitate effective and rapid prediction of sensitivity to paclitaxel is therefore an urgent medical requirement. In the present study, $G$ protein signaling modulator 2 (GPSM2) mRNA levels were significantly associated with taxane sensitivity in experiments based on the Gene Expression Omnibus (GEO) online database. Immunohistochemical analysis consistently revealed a significant association of GPSM2 protein levels with paclitaxel sensitivity in breast cancer patients. Knockdown of GPSM2 reduced the sensitivity of breast cancer cells to paclitaxel via regulation of the cell cycle. Animal experiments further corroborated our in vitro findings. These results suggest that GPSM2 plays an important role in breast cancer resistance, supporting its utility as a potential target for improving drug susceptibility in patients as well as a marker of paclitaxel sensitivity.
\end{abstract}

Correspondence to: Professor Qingjie Lv, Department of Pathology, Shengjing Hospital of China Medical University, 36 Sanhao Street, Heping, Shenyang, Liaoning 110001, P.R. China

E-mail: cmu_lvqingjie@126.com

Professor Ye Zhang, The First Laboratory of The Cancer Institute, The First Hospital of China Medical University, 155 North Nanjing Street, Shenyang, Liaoning 110001, P.R. China

E-mail: yzhang21@cmu.edu.cn

*Contributed equally

Key words: breast cancer, GPSM2, paclitaxel, cell cycle

\section{Introduction}

In China, breast cancer is one of the leading causes of cancer-related deaths in women. Systemic (i.e., neoadjuvant) chemotherapy is currently an integral part of the treatment regime for breast cancer (1). Due to the heterogeneity of the disease and individual differences, $20-30 \%$ patients subjected to neoadjuvant chemotherapy show primary resistance to chemotherapeutic drugs, which could delay the timing of local treatment and severely affect patient prognosis and quality of life $(2,3)$. Therefore, identification of markers that facilitate rapid and easy prediction of sensitivity to neoadjuvant chemotherapy is of significant value for evaluating the individual choice of treatment for patients and clarifying the mechanisms underlying resistance to chemotherapy.

Taxane is a traditional cytotoxic antitumor drug that mainly promotes polymerization of tubulin and inhibits spindle motility (4). In addition, studies have shown that taxanes affect variouc non-mitotic functions of the microtubules, such as molecular trafficking, signaling, and could kill interphase cancer cells (5-7). Taxane-based combination chemotherapy is generally the first choice for adjuvant and neoadjuvant chemotherapy of breast cancer patients $(8,9)$. However, many patients show continued resistance to taxane-based chemotherapy. Multiple molecular players are linked to taxane resistance; these include efflux pumps (10), DNA repair mechanisms (11), and microtubule alterations (12). In addition, emerging evidence indicates that both non-coding RNAs and epigenetic effectors may also be implicated in taxane resistance (7). Using the Gene Expression Omnibus (GEO) online database, G-protein signaling modulator 2 (GPSM2) was identified as a molecule significantly associated with taxane sensitivity. GPSM2 (also known as LGN) was initially shown to regulate proper positioning of the cell spindle, maintain symmetry of cell division, and play an important role in the G2/M phase involved in cell division (13-15). He and 
co-researchers demonstrated that GPSM2 is overexpressed in hepatocellular carcinoma and its expression levels are related to clinical characteristics, supporting a potential function as a tumor-suppressor gene (16). Overexpression of GPSM2 in breast cancer tissues was reported by Fukukawa et al (15). However, the possible involvement of GPSM2 in paclitaxel sensitivity has not been examined to date.

In the present study, we performed bioinformatics-based screening of the paclitaxel resistance-related gene, GPSM2, which was further confirmed in clinical specimens as well as in vitro and in vivo experiments. Our collective findings indicate that GPSM2 plays an important role in paclitaxel resistance and may thus serve as a marker of paclitaxel sensitivity in breast cancer.

\section{Materials and methods}

Bioinformatic analysis. Using the Gene Expression Omnibus database (GEO; http://www.ncbi.nlm.nih.gov/geo/) of the National Center for Biotechnology Information (NCBI) (17), raw gene expression profiles and clinical data available for breast cancer were downloaded from GSE25055 (18), GSE25065 (18) and GSE41998 (19), and data on patients receiving paclitaxel (PTX)-based neoadjuvant chemotherapy (NAC) were selected for further analyses. Univariate logistic regression (LR) was performed with the gene expression level as the independent variable and pathological complete response (pCR) status as covariates. Multiple hypothesis testing was applied on the P-value of LR for each gene, and genes with FDR $\mathrm{q}<0.25$ were identified as pCR-related. A Venn diagram was further constructed to identify pCR-related genes shared by the three datasets.

Genomic and drug sensitivity data on the NCI60 cell line was downloaded and set into the correlation analysis between each pCR-related gene with sensitivity to PTX (20). Based on the spectrum of the correlation coefficient calculated from two non-standard correlations of PTX (NSC125973 and NSC758645), the first 20 genes with higher correlation coefficients were collected into two ranking systems. The importance of each gene was quantified based on the sum of its ranking scores in the two-ranking system, and the gene with the lower value was identified as more significant.

Publicly available $\mathrm{GI}_{50}\left[-\log 10\left(\mathrm{IC}_{50}\right)\right.$, molar drug concentration for $50 \%$ growth inhibition] data on PTX (NSC125973 and NSC758645) and genomic data on the NCI60 cell line were obtained via the rcellminer R package (20). In total, 5 breast cancer (MCF7, MDA-MB-231, HS578T, BT-549 and T47D) and 7 ovarian cancer (SK-OV-3, IGROV1, OVCAR-3, OVCAR-4 and OVCAR-8) cell lines were included in the analysis. Spearman correlation was performed to confirm the correlation coefficients (r-value) between $\mathrm{GI}_{50}$ and GPSM2. The ranking lists included genes with the 20 top highest r-values for each drug (NSC125973 or NSC758645). The summed ranking of an overlapped gene in the two ranking lists was calculated, with lower values indicating higher importance of that gene.

Gene set enrichment analysis (GSEA) was performed using the JAVA program (http://www.broadinstitute.org/gsea) with GSE25055, GSE25065 or GSE41998. The MSigDB H: hallmark gene set (50 available) and C2 CP: KEGG gene set (186 available) collections were functional gene sets (21).
Expression of GPSM2 was set to annotate phenotypes. Gene sets with a FDR value $<0.25$ were considered significantly enriched. The overlapping significant gene sets among these three data sets were taken as enriched gene sets.

Clinical breast cancer samples. A total of 85 invasive ductal cancer (IDC) specimens of patients undergoing core biopsy were obtained between January 2011 and December 2014 at Shengjing Hospital of the China Medical University (Shenyang, Liaoning, China). Demographic and clinical characteristics, such as age, sex, and stage at diagnosis, were collected. This study was approved by the Ethics Committee of the China Medical University, and all patients signed informed consent, which was consistent with the Declaration of Helsinki. All patients underwent neoadjuvant chemotherapy and treatment with 2-3 cycles of the PTX regimen at Shengjing Hospital, Shengjing, China. Based on different responses to PTX, the patients were divided into four groups: Complete remission (CR) group, where the tumor disappeared and remained absent for at least 4 weeks; partial remission (PR), where the longest diameter of the tumor was reduced by $>30 \%$ or the sum of tumor diameter was reduced more than $50 \%$ and maintained more than 4 weeks; progressive disease (PD), whereby the largest diameter of the tumor increased by $>20 \%$ or the sum of the tumor diameter increased by $>25 \%$; and stable disease (SD), a stage between PR and PD, established following at least 2 cycles of chemotherapy; $\mathrm{CR}+\mathrm{PR}$ are proportional to efficiency. In a total of 85 patients, 2 patients were evaluated as having PD, 23 patients were evaluated as presenting with $\mathrm{SD}, 2$ patients were evaluated as having $\mathrm{CR}$, and 58 patients were evaluated with PR. PD and SD groups were determined as exhibiting resistance to PTX; CR and PR group were determined as being sensitive to PTX.

Cell lines and cultures. The human breast cancer cell lines MDA-MB-231, T47D and MCF-7 were purchased from the Cell Bank of the Type Culture Collection of the Chinese Academy (Shanghai, China). MDA-MB-231 cells were grown in L15 (Invitrogen; Thermo Fisher Scientific, Inc.). MCF-7 and T47D cells were grown in Dulbecco's modified Eagle's medium (DMEM; Invitrogen; Thermo Fisher Scientific, Inc.). All human breast cancer cell lines were supplemented with $10 \%$ fetal bovine serum (FBS) and maintained at $37^{\circ} \mathrm{C}$ with $5 \% \mathrm{CO}_{2}$.

Immunocytochemical staining. Paraffin sections were deparaffinized with xylene and autoclaved with sodium citrate, $\mathrm{pH}$ 6.0. Sections were incubated with the primary antibody (anti-human rabbit GPSM2; cat. no. ab84571, Abcam) at a dilution of $1: 100$ overnight at $4{ }^{\circ} \mathrm{C}$ and the secondary antibodies (1:200 diluted biotinylated goat-anti-rabbit solution) (Vector Laboratories, Inc.; cat. no. PK-4001) for $30 \mathrm{~min}$ on the following day. Next, sections were stained using 3,3'-diaminobenzidine (DAB) for microscopic observation and counterstained with hematoxylin. A semi-quantitative scoring system was adopted. The percentage of positively stained cells was scored as follows: $0,<10 \% ; 1,10-25 \% ; 2$, $26-50 \% ; 3,51-75 \%$; and $4,>75 \%$. Intensity was scored as '-' (negative), '+' (weak), '++' (moderate) and '+++' (strong). The IHC score was the percentage score multiplied by the 
staining intensity score. IHC scores $\geq 4$ were indicative of a high expression of GPSM2.

$R T-q P C R$. Reverse transcription polymerase chain reaction (RT-PCR) array was used to analyze GPSM2 expression or efficiency of transfection. RT-qPCR was performed according to our previously reported method (22). The primer sequences for amplification were: Forward (5'-AGCCAGTCGGTACTT AGCCA-3') and reverse (5'-TTGTGGTAGCAGGTGGTG GA-3') for GPSM2; and forward (5'-GGTGAAGGTCGGAGT CAACGG-3') and reverse (5'-GAGGTCAATGAAGGGGTC ATTG-3') for the $18 \mathrm{~S}$ control. PCR conditions were as follows: $95^{\circ} \mathrm{C}$ for $5 \mathrm{~min}, 95^{\circ} \mathrm{C}$ for $15 \mathrm{sec}, 62.8^{\circ} \mathrm{C}$ for $15 \mathrm{sec}, 72^{\circ} \mathrm{C}$ for $20 \mathrm{sec}$ and $72^{\circ} \mathrm{C}$ for $5 \mathrm{~min}$ for 35 cycles. Relative expression of mRNA was calculated via the comparative cycle threshold method using $18 \mathrm{~S}$ as a reference.

Western blot analysis. Cells were extracted and proteins was lysed in RIPA lysis buffer. Equivalent amounts (40 $\mu \mathrm{g})$ of protein were isolated by $10 \%$ SDS-PAGE (Bio-Rad) and transferred to polyvinylidene fluoride (PVDF) membranes (Millipore). Membranes were blocked with 5\% non-fat milk for $40 \mathrm{~min}$ and incubated with primary antibodies: Anti-GPSM2 (dilution 1:1,000, Abcam) and anti- $\beta$-actin (cat. no. sc-47778; dilution 1:1,000; Santa Cruz Biotechnology) overnight at $4^{\circ} \mathrm{C}$. Following further washing in TTBS, membranes were incubated with secondary antibodies for $40 \mathrm{~min}$. After extensive washing, chemiluminescence was detected using the ECL kit (Millipore). Image J 6.0 software (NIH, National Institutes of Health, Bethesda, MD, USA) was used to analyze the images.

Cell proliferation assay. Cell viability was determined by 1-(4,5-demethylthiazol-2-yl)-3,5-diphenyltetrazolium bromide (MTT) assay. Cells were cultured in 96-well plates and treated with different concentrations of PTX for $48 \mathrm{~h}$. After incubation with MTT $(0.5 \mathrm{mg} / \mathrm{ml})$ for $4 \mathrm{~h}$, the supernatant was discarded and $200 \mu 1$ DMSO was added to each well. The absorbance at $570 \mathrm{~nm}$ was detected spectrophotometrically.

Cell transfection and treatments. Cell transfection was performed according to the small interfering RNA (siRNA) sequence transfection protocol for Lipofectamine 2000 (Invitrogen; Thermo Fisher Scientific, Inc.). The sequence of the GPSM2-specific siRNA was 5'-GGUAAUCUGGGA AACACCU-3'. Nonsense RNAi (5'-UUCUCCGAACGU GUC ACGUTT-3') was used as a negative control (NC). At $48 \mathrm{~h}$ after transfection, the transfection efficiency of GPSM2 was identified by RT-qPCR and western blot analyses.

Colony formation assay. For the colony formation assay, transfected MDA-MB-231 cells were harvested and seeded at a density of 500 cells per well in 12 -well plates at $37^{\circ} \mathrm{C}$ and $5 \% \mathrm{CO}_{2}$ in a humidified incubator for 2 weeks. Results were determined and images obtained under a microscope (magnification x100) following Giemsa staining. The colony numbers in each well were calculated.

Transfection of the GPSM2 WT plasmid. GPSM2 wild-type (WT) plasmid and control plasmid were purchased from Genechem (Shanghai, China). Cells were seeded at $4 \times 10^{5}$ cells
Table I. Genes with r-values $>0.4$ in NSC125973 and NSC758645.

\begin{tabular}{|c|c|c|c|}
\hline \multicolumn{2}{|c|}{ NSC125973 } & \multicolumn{2}{|c|}{ NSC75864 } \\
\hline Gene symbol & r-value & Gene symbol & r-value \\
\hline$M E X 3 C$ & 0.661 & USP13 & 0.757 \\
\hline WASF 1 & 0.61 & SREKIIPI & 0.658 \\
\hline$L M N B 2$ & 0.556 & $W B P 1 L$ & 0.618 \\
\hline$O B F C 1$ & 0.516 & $N F I B$ & 0.609 \\
\hline NUP160 & 0.516 & TCTN1 & 0.577 \\
\hline LRPAPI & 0.506 & ARL3 & 0.571 \\
\hline XRCC4 & 0.459 & ACTL6A & 0.556 \\
\hline$W B P 1 L$ & 0.454 & XRCC4 & 0.538 \\
\hline LSM6 & 0.444 & SLC39A6 & 0.521 \\
\hline SREKIIP1 & 0.43 & AKAP10 & 0.508 \\
\hline IPO5 & 0.428 & TSPAN31 & 0.497 \\
\hline GPSM2 & 0.427 & ZERI & 0.494 \\
\hline \multirow[t]{11}{*}{$C D C 40$} & 0.414 & $O B F C 1$ & 0.49 \\
\hline & & SMC4 & 0.479 \\
\hline & & $H A G H$ & 0.475 \\
\hline & & $H 2 A F Y 2$ & 0.435 \\
\hline & & $A F F 3$ & 0.426 \\
\hline & & $A B C F 1$ & 0.418 \\
\hline & & HIFIAN & 0.417 \\
\hline & & $P I G Q$ & 0.415 \\
\hline & & $M L P H$ & 0.409 \\
\hline & & GPSM2 & 0.402 \\
\hline & & CCNE2 & 0.401 \\
\hline
\end{tabular}

$M E X 3 C$, mex-3 RNA binding family member C; WASF1, WASP family member 1; $L M N B 2$, lamin $\mathrm{B} 2$; $O B F C 1$, oligonucleotide/oligosaccharide-binding fold containing 1; NUP160, nucleoporin 160; $L R P A P 1, \mathrm{LDL}$ receptor related protein associated protein 1; XRCC4, $\mathrm{X}$-ray repair cross complementing 4 ; WBPIL, WW domain binding protein 1 like; LSM6, LSM6 homolog; SREK1IP1, splicing regulatory glutamine/lysine-rich protein 1 interacting protein 1; IPO5, importin 5; GPSM2, G protein signaling modulator 2; $C D C 40$, cell division cycle 40 ; USP13, ubiquitin specific peptidase $13 ; N F I B$, nuclear factor I B; TCTN1, tectonic family member 1; ARL3, ADP ribosylation factor like GTPase 3; ACTL6A, actin like 6A; SLC39A6, solute carrier family 39 member 6 ; AKAP10, A-kinase anchoring protein 10; TSPAN31, tetraspanin 31; ZER1, zyg-11 related cell cycle regulator; $S M C 4$, structural maintenance of chromosomes 4; $H A G H$, hydroxyacylglutathione hydrolase; $H 2 A F Y 2, \mathrm{H} 2 \mathrm{~A}$ histone family member Y2; $A F F 3$, AF4/FMR2 family member 3; $A B C F 1$, ATP binding cassette subfamily $\mathrm{F}$ member 1 ; HIFIAN, hypoxia inducible factor 1 subunit $\alpha$ inhibitor; $P I G Q$, phosphatidylinositol glycan anchor biosynthesis class Q; $M L P H$, melanophilin; CCNE2, cyclin E2.

per well in 6-well plates overnight and transfected by Lipofectamine 2000 reagent with the GPSM2 WT plasmid. Forty-eight hours after transfection, GPSM2 expression was evaluated using western blot analysis.

Cell cycle assay. Propidium iodide (PI) staining was used to detect the cell cycle. Cells in the log phase were trypsinized, 
A

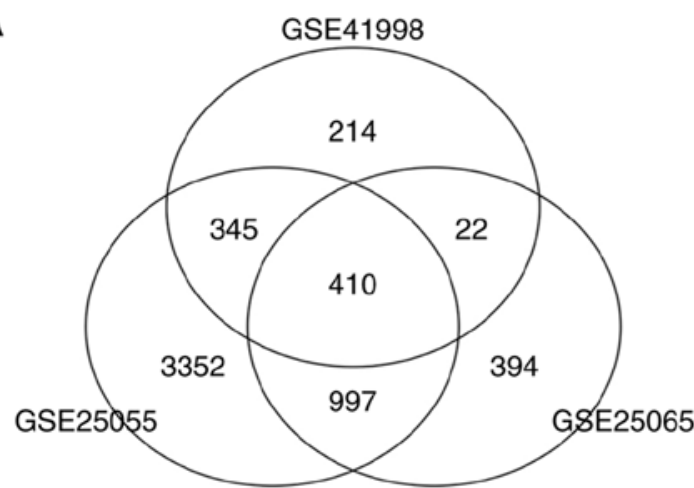

C

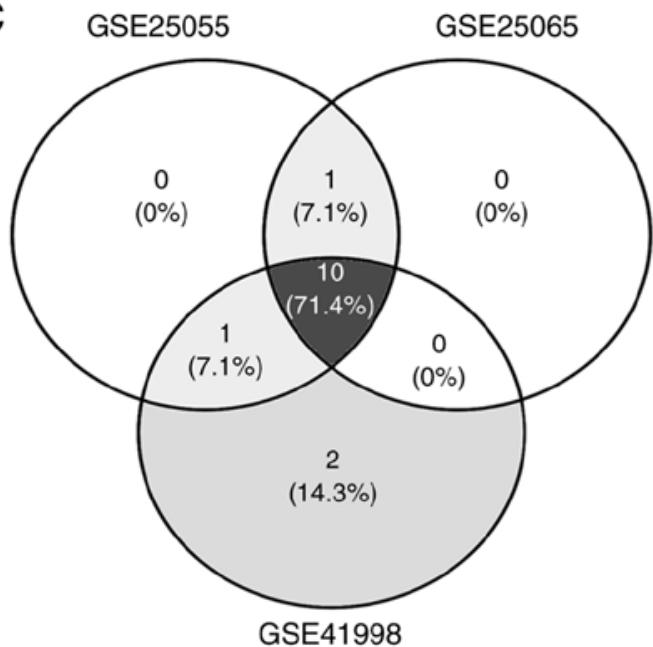

B

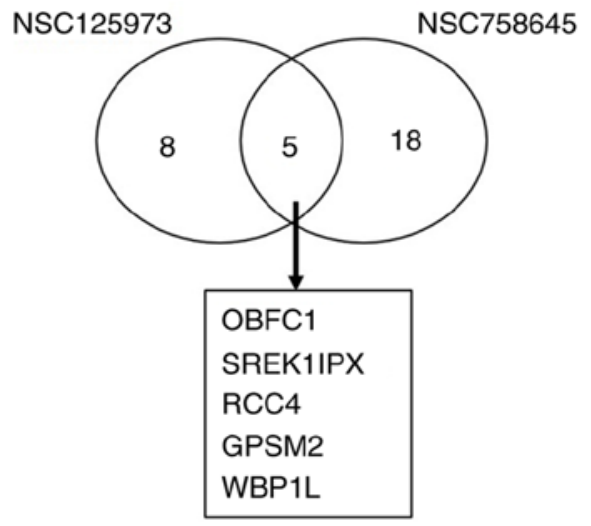

D

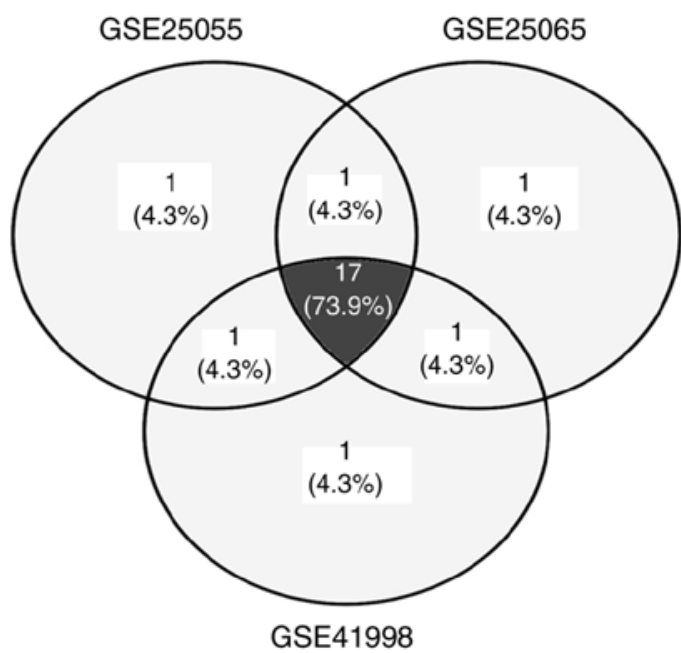

E
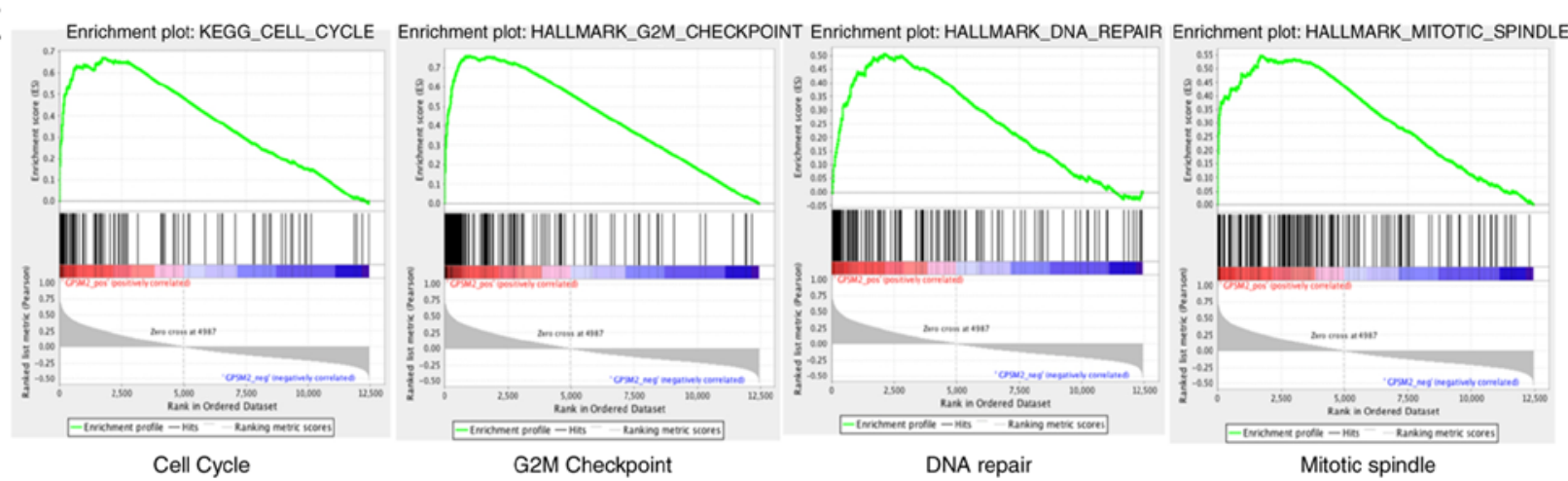

Figure 1. Online database screening for relevant genes closely related to paclitaxel primary drug resistance. (A) Venn plot showing 410 genes shared by the three GEO datasets. (B) Venn plot showing 5 genes shared by NSC125973 and NSC758645. (C) Significant gene sets with GPSM2 high-expression phenotypes when hallmarks were used as a reference. (D) Significant gene sets with GPSM2 high-expression phenotypes when KEGG signaling pathways were used as a reference. (E) Overlapping gene sets from the two above GSEA analyses. KEGG, Kyoto Encyclopedia of Genes and Genomes; OBFC1, oligonucleotide/oligosaccharide-binding fold containing 1; SREK1IP1, splicing regulatory glutamine/lysine-rich protein 1 interacting protein 1; RCC4, regulator of chromosome condensation 1; GPSM2, G protein signaling modulator 2; WBP1L, WW domain binding protein 1 like.

centrifuged at $300 \times \mathrm{g}$ for $5 \mathrm{~min}$, and washed with PBS. Next, the sediment was suspended with $400 \mathrm{ml}$ PBS with $50 \mathrm{mg} / \mathrm{ml} \mathrm{PI}$ and $100 \mathrm{mg} / \mathrm{ml}$ RNase at $37^{\circ} \mathrm{C}$ for $30 \mathrm{~min}$. Flow cytometry (BD AccuriTM C6 Flow cytometer) was used to determine the percentages of cells in the G1, S, and G2 phases, and the data were analyzed by WinMDI version software (http://www.cyto.purdue.edu/flowcyt/software/Winmdi.htm).

Generation of stable GPSM2-knockdown cell line. GPSM2 shRNA lentiviral particles were obtained from Genechem
Technology Corp., Ltd. (Shanghai, China). MDA-MB-231 cells were infected with GPSM2 shRNA lentiviral particles following the manufacturer's instructions.

Animal experiments. Male athymic nude mice (BALB/c-nu/nu) (4-6 weeks old, 18-24 g) were purchased from Shanghai Slike Experimental Animals Co. (Shanghai, China) and housed under specific pathogen-free conditions. The maintenance conditions for the mice were as follows: temperature, $23-27^{\circ} \mathrm{C}$; humidity, 40-60\%; ventilation, 15 times/h; $12 \mathrm{~h}$ light/12 h dark 
A

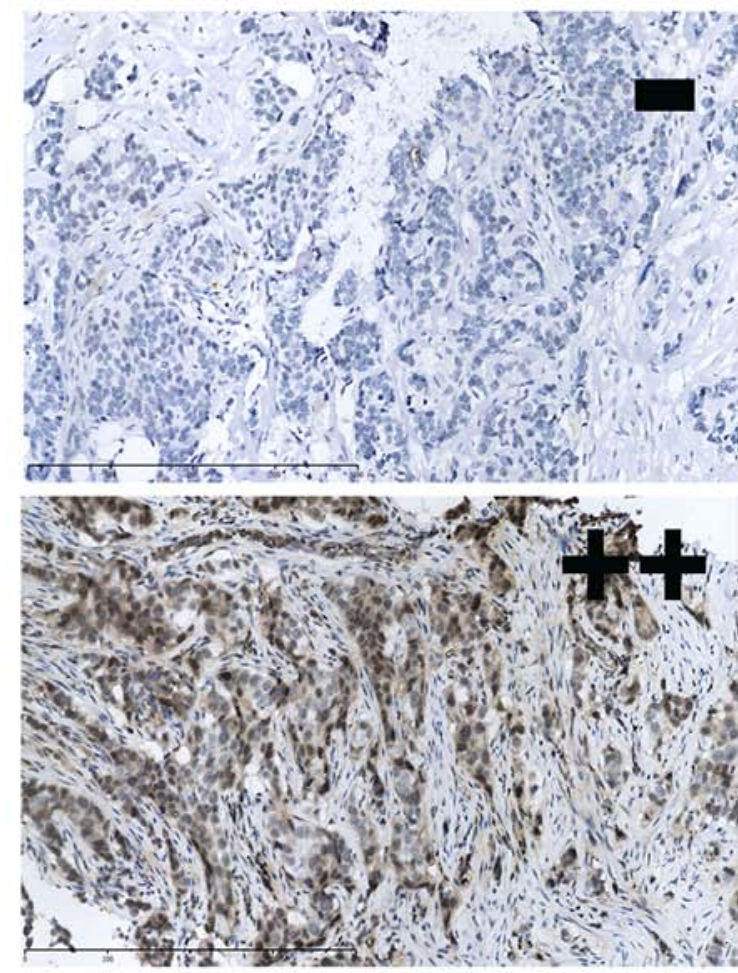

B

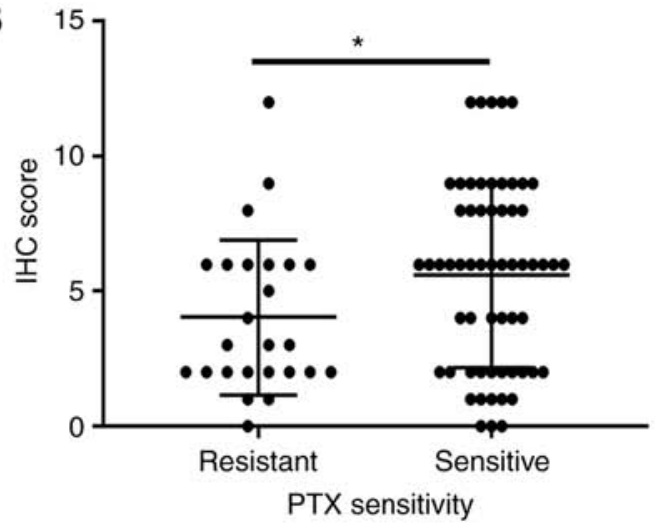

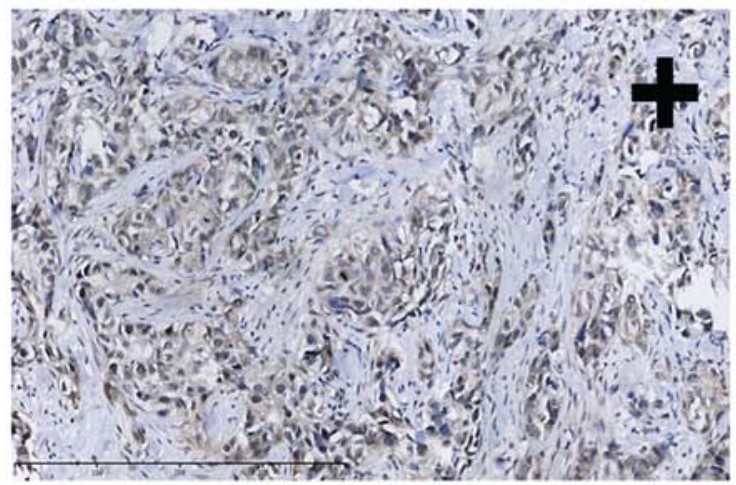

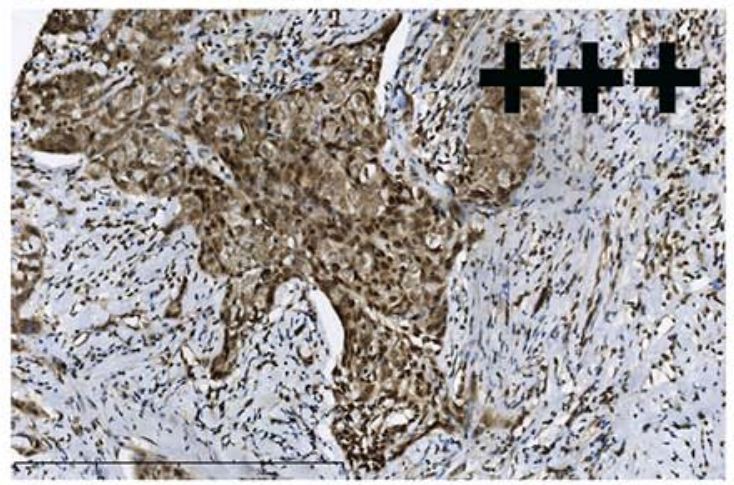

C

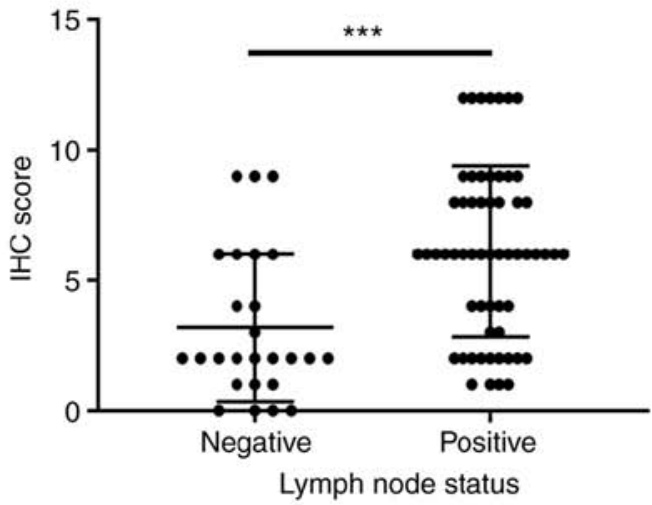

Figure 2. High GPSM2 protein expression is valuable to predict resistance to PTX in breast cancer patients. (A) Representative images of GPSM2 staining in human breast tissue samples: '-' (negative staining intensity), '+' (weak staining intensity), '++' (moderate staining intensity), and '+++' (strong staining intensity) (Scale bar, $300 \mu \mathrm{m}$ ). (B) IHC scores of GPSM2 were significantly reduced in PTX-resistant tissues relative to PTX-sensitive tissues ( $\left.{ }^{*}<0.05\right)$. (C) IHC scores of GPSM2 were significantly reduced in patients with lymph node-negative status, compared to those with lymph node-positive status $\left(^{* * *} \mathrm{P}<0.05\right)$. $\mathrm{PTX}$, paclitaxel; GPSM2, G protein signaling modulator 2; IHC, immunohistochemistry.

cycle. The mice were fed standard laboratory food and water. MDA-MB-231 cells $\left(1 \times 10^{7}\right)$ was directly injected into the exposed no. 4 inguinal mammary pad of each mouse. Tumor volume was calculated as: Volume $=\left(\right.$ width $^{2} \mathrm{x}$ length $) / 2$. Paclitaxel (PTX) (10 mg/kg once every 3 days) was administered via i.p. injection starting from the time the tumor volume reached $\sim 30 \mathrm{~mm}^{3}$. The health and maintenance condition of the mice were monitored every 3 days. After 2 weeks, the mice were sacrificed by cervical dislocation and the tumors were removed and weighed. The maximum diameter of a single tumor found was $12 \mathrm{~mm}$ and no mouse developed multiple tumors. All mice were in a good body condition throughout the experiment. All animal experiments conformed to the Guide for Care and Use of Laboratory Animals and were approved by the Animal Care and Use Committee of China Medical University.
Statistical analysis. Statistical significance was examined using the Student's t test using the SPSS 13.0 software package (SPSS, Inc.). Growth cell data were compared using one-way analysis of variance (ANOVA) and Tukey's multiple comparisons test. Correlations between two variables were evaluated with Spearman's rank correlation coefficient. For all statistical analyses, the level of significance was set at $\mathrm{P}<0.05$.

\section{Results}

Online database analysis to screen the relevant genes closely related to paclitaxel primary drug resistance. A total of 306, 182 and 121 patients were included in the GSE25055 (18), GSE25065 (18) and GSE41998 (19) datasets, respectively. Logistic regression analyses showed that 5,104, 1,823 and 
Table II. Correlations between GPSM2 expression and clinical characteristics of the breast cancer patients in the present study $(\mathrm{N}=85)$.

\begin{tabular}{|c|c|c|c|c|}
\hline \multirow[b]{2}{*}{ Characteristics } & \multirow[b]{2}{*}{ Cases (n) } & \multicolumn{2}{|c|}{ GPSM2 expression } & \multirow[b]{2}{*}{ P-value } \\
\hline & & Positive n (\%) & Negative $\mathrm{n}(\%)$ & \\
\hline Age (years) & & & & 0.377 \\
\hline$\leqq 45$ & 27 & $19(70.37)$ & $8(26.63)$ & \\
\hline$>45$ & 58 & 35 (60.34) & $23(39.66)$ & \\
\hline Clinical stage & & & & $0.016^{\mathrm{a}}$ \\
\hline I-II & 43 & $22(51.16)$ & $21(48.84)$ & \\
\hline III-IV & 42 & $32(76.19)$ & $10(23.81)$ & \\
\hline Lymph node status & & & & $0.001^{\mathrm{a}}$ \\
\hline Negative & 26 & $10(38.46)$ & $16(61.54)$ & \\
\hline Positive & 59 & $44(74.58)$ & $15(25.42)$ & \\
\hline Tumor size $(\mathrm{cm})$ & & & & 0.474 \\
\hline$<5$ & 59 & $36(61.01)$ & $23(38.98)$ & \\
\hline$\geqq 5$ & 26 & $18(69.23)$ & $8(30.77)$ & \\
\hline ER & & & & 0.349 \\
\hline Negative & 31 & $22(70.97)$ & $9(29.03)$ & \\
\hline Positive & 54 & $33(61.11)$ & $21(38.89)$ & \\
\hline PR & & & & 0.547 \\
\hline Negative & 31 & $22(70.97)$ & $9(29.03)$ & \\
\hline Positive & 54 & $34(62.96)$ & $22(40.74)$ & \\
\hline Her2 & & & & 0.138 \\
\hline Negative & 42 & $30(71.43)$ & $12(28.57)$ & \\
\hline Positive & 43 & $24(55.81)$ & $19(44.19)$ & \\
\hline PTX sensitivity & & & & $0.015^{\mathrm{a}}$ \\
\hline Resistant & 25 & $11(44.00)$ & $14(56.00)$ & \\
\hline Sensitive & 60 & $43(71.67)$ & $17(28.33)$ & \\
\hline
\end{tabular}

GPSM2, G protein signaling modulator 2; ER, estrogen receptor; PR, progesterone receptor; Her2, human epidermal growth factor receptor 2; PTX, paclitaxel. aSignificant difference.

991 genes were statistically significant $(\mathrm{P}<0.05$, FDR $<0.25)$ in the three respective datasets. In the Venn plot, 410 genes were shared by the three datasets (Fig. 1A), which were pCR-related. In NCI-60 breast and ovarian cancer datasets, the correlation of each pCR-related gene with drug sensitivity to NSC125973 and NSC758645 was ranked. Genes with r-values $>0.4$ in NSC125973 and NSC758645 are depicted in Table I. GPSM2 was determined to be an overlapped gene (Fig. 1B). To further investigate the pathways regulated by GPSM2, gene set enrichment analysis was applied. Ten gene sets showed significant GPSM2 high-expression phenotypes when hallmarks were used as the reference in this step (Fig. 1C). Seventeen gene sets showed significant GPSM2 high-expression phenotype when Kyoto Encyclopedia of Genes and Genomes (KEGG) signaling pathways were used as the reference (Fig. 1D). The overlapping gene sets are listed in Fig. 1E. Cell cycle-related gene sets ('HALLMARK_G2M_CHECKPOINT', 'HALLMARK_MITOTIC_SPINDLE', 'KEGG_CELL_ CYCLE' and 'KEGG_DNA_REPLICATION', among others) were significantly associated with the GPSM2 high-expression phenotype.
GPSM2 is significantly correlated with paclitaxel sensitivity in breast cancer patients. To confirm the clinical significance of GPSM2 in breast cancer, we selected 85 patients undergoing neoadjuvant chemotherapy and performed immunohistochemical analysis of core biopsy pathological sections. Representative examples of each level of staining are shown in Fig. 2A. The GPSM2 staining score was significantly higher in the PTX-sensitive patients (Fig. 2B). Moreover, the staining score was significantly increased in patients with a positive lymph node status (Fig. 2C). Based on the IHC results, staining was quantified and classified into positive and negative groups. Data from the Chi-square test showed significant correlations between GPSM2 expression and lymph node status $(\mathrm{P}=0.001)$, TNM stage $(\mathrm{P}=0.016)$ and $\mathrm{PTX}$ sensitivity $(\mathrm{P}=0.015)$ (Table II). The IHC score of GPSM2 was higher in advanced-stage cancer patients (clinical stage III-IV) than in early-stage cancer patients (clinical stage I-II) $(6.381 \pm 3.450$, $\mathrm{n}=42$ vs. $4.093 \pm 3.030, \mathrm{n}=43$, respectively, $\mathrm{P}=0.002)$. In additional, the rate of PTX-resistance in advanced cancer was $13 / 42(30.95 \%)$, while the rate of PTX-resistance in early-stage cancer was $12 / 43(27.90 \%)$. Chi-square test indicated that the 


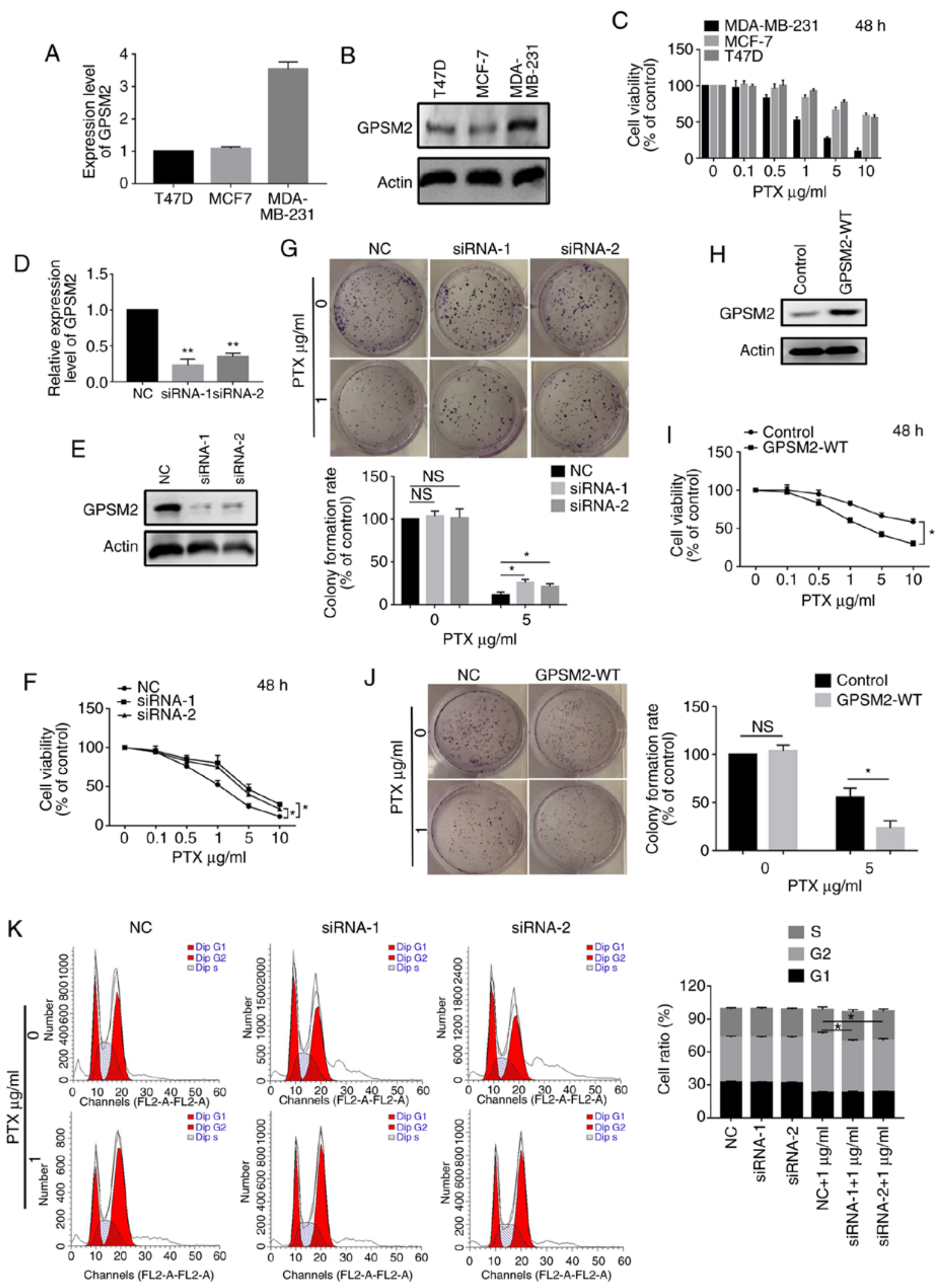

Figure 3. Knockdown of GPSM2 increases resistance to PTX in breast cancer MDA-MB-231 cells. (A and B) GPSM2 mRNA and protein expression in three breast cancer cell lines (T47D, MCF7 and MDA-MB-231). (C) Cell proliferation assays were performed following treatment with different concentrations $(0,0.1,0.5,1,5$ and $10 \mu \mathrm{g} / \mathrm{ml}$ ) of PTX in the three breast cancer cell lines. (D and E) MDA-MB-231 cells were transfected with either GPSM2-siRNA or NC-siRNA and GPSM2 mRNA and protein expression levels were detected via RT-qPCR and western blot analyses, respectively. ${ }^{* *} \mathrm{P}<0.01$ vs. the NC group. (F) Cell viability assay showing that GPSM2 knockdown increased the resistance of MDA-MB-231 cells to PTX. "P<0.05. (G) After treatment with PTX, the number of colonies formed by MDA-MB-231 cells transfected with NC siRNA was lower than those formed by cells transfected with GPSM2 siRNA. $\mathrm{P}<0.05$; ns, not significant. (H) MDA-MB-231 cells were transfected with either GPSM2-WT or empty vector plasmid and GPSM2 protein expression was detected via western blot analyses. (I) Cell viability assay showing that GPSM2 overexpression decreased the resistance of MDA-MB-231 cells to PTX. " P<0.05. (J) After treatment with PTX, the number of colonies formed by MDA-MB-231 cells transfected with the empty vector plasmid was higher than the number formed by cells transfected with the GPSM2-WT plasmid. "P<0.05; ns, not significant. (K) GPSM2 affects the activity of PTX to trigger G2 phase arrest in breast cancer. GPSM2 knockdown reduced paclitaxel-induced G2 phase arrest. "P<0.05. PTX, paclitaxel; GPSM2, G protein signaling modulator 2; NC, negative control. 
A

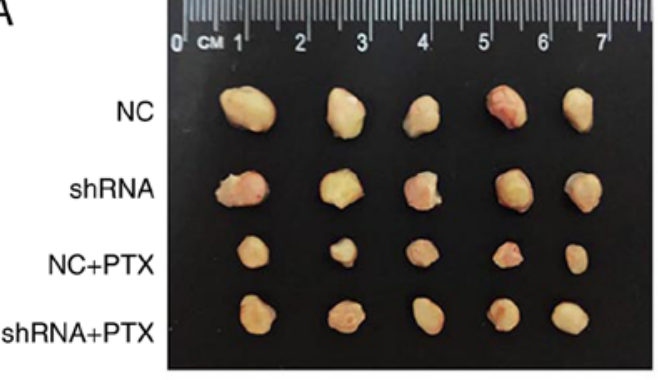

B

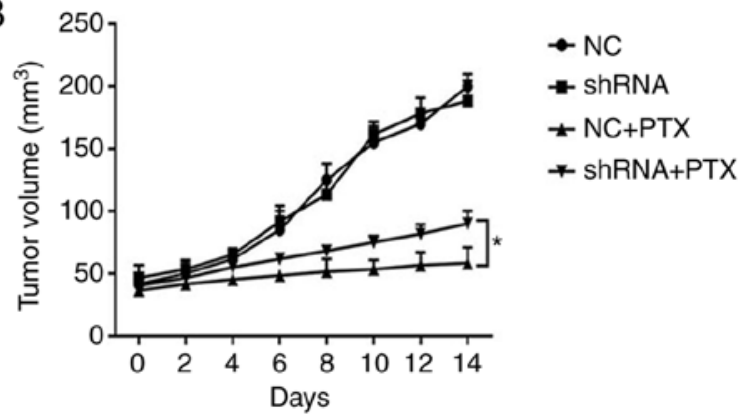

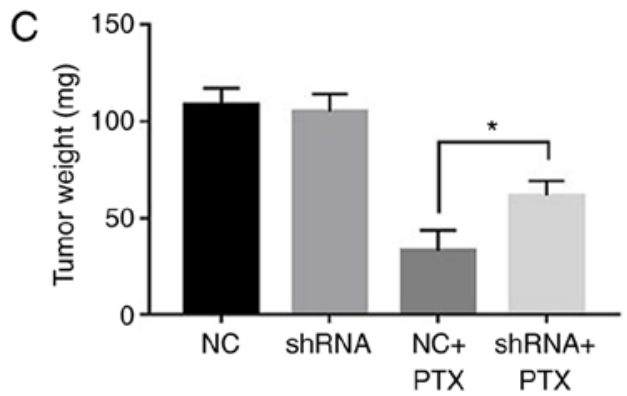

Figure 4. Combination treatment with GPSM2 shRNA and PTX in a nude mouse model. (A) Representative images of tumors isolated from each group after inoculation of MDA-MB-231 cells exposed to the different treatments (NC, shRNA, NC+PTX and shRNA+PTX). (B) Tumor volumes in orthotopic implantation model mice. (C) Weights of tumors in orthotopic implantation model mice. "P<0.05. PTX, paclitaxel; GPSM2, G protein signaling modulator 2; NC, negative control.

resistance of PTX had no significance associated with TNM stage $(\mathrm{P}=0.758)$. Based on our results, advanced-stage cancer showed higher GPSM2 scores, but was not associated with better clinical benefit.

Knockdown of GPSM2 expression reduces the sensitivity of breast cancer cells to paclitaxel. Next, we determined the mRNA and protein levels of GPSM2 in three breast cancer cell lines (MDA-MB-231, T47D, MCF7). Interestingly, MDA-MB-231 cells expressed considerably higher levels of GPSM2 than the other cell lines (Fig. 3A and B). Cell proliferation assays were performed after the cells were treated with different concentrations of PTX $(0,0.1,0.5,1,5$ and $10 \mu \mathrm{g} / \mathrm{ml})$. MDA-MB-231 cells with relatively high GPSM2 expression tended to have significantly higher sensitivity values (Fig. 3C). Accordingly, this cell line was selected for further investigation. The simplicity of small interfering RNA (siRNA) manufacturing and transient nature of the effect per dose are suited for cell assay (23). Thus, to ascertain the involvement of GPSM2 in PTX sensitivity, knockdown of GPSM2 expression was performed using specific siRNAs, and transfection efficiency was assessed via RT-qPCR (Fig. 3D) and western blot analyses (Fig. 3E). Notably, depletion of GPSM2 led to lower PTX sensitivity in the MDA-MB-231 cells (Fig. 3F). Moreover, the GPSM2 siRNA-transfected groups displayed more colonies than those transfected with NC-siRNA after PTX treatment (Fig. 3G). MCF-7 cells were selected for the subsequent experiments because of the low expression level of GPSM2. Overexpression of GPSM2 was performed using the GPSM2-WT plasmid, and transfection efficiency was assessed via western blot analyses (Fig. 3H). Notably, overexpression of GPSM2 led to significantly higher PTX sensitivity in MCF-7 cells (Fig. 3I). Moreover, GPSM2-WT plasmid transfected groups displayed less colonies than those transfected with the empty vector plasmid after PTX treatment (Fig. 3J). PTX-treated cells are known to undergo G2 phase arrest. Further GSEA analyses revealed that GPSM2 is significantly correlated with cell cycle-related gene sets. Accordingly, we assessed whether GPSM2 affects the activity of PTX in triggering G2 phase arrest in breast cancer cells. Knockdown of GPSM2 clearly reduced PTX-induced G2 phase arrest (Fig. 3K). PTX induced cell cycle arrest of $12.92 \pm 0.510 \%$ of cells in the G2 phase, whereas only $5.667 \pm 0.667$ of cells were arrested in the $\mathrm{G} 2$ phase after knockdown of GPSM2.

GPSM2 influences paclitaxel sensitivity in vivo. To further ascertain the potential effects of GPSM2 on paclitaxel sensitivity, we used an orthotopic implantation mouse model of MDA-MB-231 cells. The orthotopic implantation mouse model lasted 14 days. Sustainable characteristic of shRNA could provide long-lasting knockdown effect (21). Thus, stable GPSM2-knockdown cells were generated using shRNA. Compared to the mice implanted with NC shRNA-transfected MDA-MB-231 cells, no significant differences in tumor growth were observed in the GPSM2 shRNA-transfected group. However, differences were evident upon treatment of both groups with PTX. Specifically, following PTX treatment, the tumor volumes of mice in the GPSM2 shRNA group were significantly increased, compared with those in the NC shRNA group $(\mathrm{P}<0.05)$ (Fig. 4A and $\mathrm{B})$. Moreover, under conditions of knockdown of GPSM2, sensitivity of breast cancer cells to PTX was reduced and tumor weights were higher (Fig. 4C).

\section{Discussion}

Breast cancer is the most common malignancy in women worldwide (1). Neoadjuvant and conventional postoperative 
chemotherapy strategies have been established as the most successful treatments to date, among which Taxol is one of the most effective drugs $(2,24)$. Owing to disease heterogeneity and individual differences, approximately 20-30\% patients subjected to neoadjuvant chemotherapy are resistant to paclitaxel (25). Thus, identification of markers that can rapidly and easily predict sensitivity to neoadjuvant chemotherapy is currently an urgent medical requirement.

Based on clinical information and gene expression profiles extracted from the Gene Expression Omnibus (GEO) (GSE25055, GSE25065 and GSE41998) database, we observed significance differences in G-protein signaling modulator 2 (GPSM2) expression in correlation with paclitaxel resistance. This finding was further validated with NSC125973, NSC758645 and GSEA analyses. GPSM2 plays an important role in controlling spindle orientation and positioning in the context of asymmetric cell division (26,27). Bioinformatics analyses in our study support a novel relationship between GPSM2 and paclitaxel resistance in breast cancer, which provides a guiding framework for subsequent experiments.

GPSM2 has also been shown to participate in neuroblast division and polarization of the apical complex. Expression of GPSM2 is closely related to progression of hepatocellular carcinoma and renal cell carcinoma (28). A previous study reported an association of GPSM2 overexpression with occurrence of breast cancer (15). However, its potential involvement in paclitaxel resistance in breast cancer patients is yet to be established. To the best of our knowledge, this is the first study to demonstrate that GPSM2 is widely downregulated in tissue samples of paclitaxel-resistant breast cancer patients and is correlated with lymph node status and clinicopathological parameters. In view of our experimental results, we propose that GPSM2 may serve as an effective indicator of paclitaxel drug sensitivity in breast cancer patients.

Drug resistance in tumor cells is an important contributory factor to disease progression (29). Here, we examined the potential role of GPSM2 expression in breast cancer by silencing its expression. In cell viability and colony formation assays, depletion of GPSM2 expression in MDA-MB-231 cells led to increased resistance to paclitaxel, supporting the hypothesis that GPSM2 is a potential drug-resistance gene in breast cancer. In view of the GSEA enrichment data showing that GPSM2 is related to the cell cycle, a flow cytometry experiment was conducted, which revealed a decrease in paclitaxel-induced G2 arrest of MDA-MB-231 cells following the silencing of GPSM2. G2 phase arrest is an important mechanism for the paclitaxel-mediated effect on cell proliferation (5). Paclitaxel, as a microtubule stabilizing agent, induces cell cycle arrest in the G2 phase and produces abnormal mitotic spindles (30). This suggests that G2 phase arrest and microtubule regulation may be the potential mechanisms of GPSM2. The association between GPSM2 and paclitaxel resistance was further validated through in vivo experiments in nude mice. Several studies have shown that GPSM2 acts as a guanylate dissociation inhibitor (GDI) to inhibit the release of G $\alpha$ from GDP, resulting in a decrease in the availability of Ga-GTP (31). In addition, paclitaxel was found to reverse the effect of GTP hydrolysis, preventing microtubules from depolymerizing $(32,33)$. The loss of GPSM2 may increase the G $\alpha$-GTP level, and then the ability of paclitaxel to induce cycle arrest by inhibiting GTP activity may decline, leading to drug resistance in patients.

Our preliminary results clearly indicate that GPSM2 is closely related to drug resistance in breast cancer. However, it should be noted that samples in this study were acquired retrospectively. Further comprehensive analyses are necessary to validate the clinical applicability of GPSM2 as a potential biomarker.

Data from the current study support a critical role of GPSM2 in breast cancer resistance. We propose that GPSM2 may serve as a potential target for improving drug susceptibility in patients as well as a marker of paclitaxel sensitivity.

\section{Acknowledgements}

Not applicable.

\section{Funding}

This study was supported by Shenyang Special Funds of Technology Innovation Plan Projects (no. F14-231-1-46), Liaoning Science and Technology Plan Projects (no. 2013225021) and Liaoning Natural Science Foundation of 2018 (no. 20180530050).

\section{Availability of data and materials}

All data generated or analyzed during the present study are included in this published article.

\section{Authors' contributions}

QL and YZ conceived and designed the experiments. ZZhang, ZL, MD, BL, XX and ZZhao performed all the experiments. ZZhang,ZL and MD analyzed the data. ZZhang and MD wrote the manuscript. All authors read and approved the manuscript and agree to be accountable for all aspects of the research in ensuring that the accuracy or integrity of any part of the work are appropriately investigated and resolved.

\section{Ethics approval and consent to participate}

All of the animal experiments conformed to the Guide for Care and Use of Laboratory Animals and were approved by the Animal Care and Use Committee of China Medical University (No. 2018113). All patients signed informed consent, which was consistent with the Declaration of Helsinki.

\section{Patient consent for publication}

Not applicable.

\section{Competing interests}

The authors declare no competing interests.

\section{References}

1. Harbeck N and Gnant M: Breast cancer. Lancet 389: 1134-1150, 2017.

2. Pondé NF, Zardavas D and Piccart M: Progress in adjuvant systemic therapy for breast cancer. Nat Rev Clin Oncol 16: 27-44, 2019. 
3. DeMichele A, Yee D and Esserman L: Mechanisms of resistance to neoadjuvant chemotherapy in breast cancer. N Engl J Med 377: 2287-2289, 2017

4. Jordan MA and Wilson L: Microtubules as a target for anticancer drugs. Nat Rev Cancer 4: 253-265, 2004.

5. Weaver BA: How Taxol/paclitaxel kills cancer cells. Mol Biol Cell 25: 2677-2681, 2014.

6. Herbst RS and Khuri FR: Mode of action of docetaxel-a basis for combination with novel anticancer agents. Cancer Treat Rev 29: 407-415, 2003.

7. Pucci P, Rescigno P, Sumanasuriya S, de Bono J and Crea F: Hypoxia and noncoding rnas in taxane resistance. Trends Pharmacol Sci 39: 695-709, 2018.

8. Palmieri C, Krell J, James CR, Harper-Wynne C, Misra V, Cleator S and Miles D: Rechallenging with anthracyclines and taxanes in metastatic breast cancer. Nat Rev Clin Oncol 7: 561-574, 2010.

9. Turner N, Biganzoli L and Di Leo A: Continued value of adjuvant anthracyclines as treatment for early breast cancer. Lancet Oncol 16: e362-e369, 2015

10. Vaidyanathan A, Sawers L, Gannon AL, Chakravarty P, Scott AL, Bray SE, Ferguson MJ and Smith G: ABCB1 (MDR1) induction defines a common resistance mechanism in paclitaxel- and olaparib-resistant ovarian cancer cells. Br J Cancer 115: 431-441, 2016.

11. Poruchynsky MS, Komlodi-Pasztor E, Trostel S, Wilkerson J, Regairaz M, Pommier Y, Zhang X, Kumar Maity T, Robey R, Burotto M, et al: Microtubule-targeting agents augment the toxicity of DNA-damaging agents by disrupting intracellular trafficking of DNA repair proteins. Proc Natl Acad Sci USA 112: 1571-1576, 2015

12. Yang J, Yu Y, Liu W, Li Z, Wei Z and Jiang R: Microtubule-associated protein tau is associated with the resistance to docetaxel in prostate cancer cell lines. Res Rep Urol 9: 71-77, 2017.

13. Du Q, Taylor L, Compton DA and Macara IG: LGN blocks the ability of NuMA to bind and stabilize microtubules. A mechanism for mitotic spindle assembly regulation. Curr Biol 12 : 1928-1933, 2002.

14. Mochizuki N, Cho G, Wen B and Insel PA: Identification and cDNA cloning of a novel human mosaic protein, LGN, based on interaction with $\mathrm{G}$ alpha i2. Gene 181: 39-43, 1996.

15. Fukukawa C, Ueda K, Nishidate T, Katagiri T and Nakamura $Y$ Critical roles of LGN/GPSM2 phosphorylation by PBK/TOPK in cell division of breast cancer cells. Genes Chromosomes Cancer 49: 861-872, 2010

16. He XQ, Zhang YF, Yu JJ, Gan YY, Han NN, Zhang MX, Ge W, Deng JJ, Zheng YF and Xu XM: High expression of G-protein signaling modulator 2 in hepatocellular carcinoma facilitates tumor growth and metastasis by activating the PI3K/AKT signaling pathway. Tumour Biol 39: 1-10, 2017.

17. Barrett T, Troup DB, Wilhite SE, Ledoux P, Rudnev D, Evangelista C, Kim IF, Soboleva A, Tomashevsky M, Marshall KA, et al: NCBI GEO: Archive for high-throughput functional genomic data. Nucleic Acids Res 37: D885-D890, 2009.

18. Hatzis C, Pusztai L, Valero V, Booser DJ, Esserman L, Lluch A, Vidaurre T, Holmes F, Souchon E, Wang H, et al: A genomic predictor of response and survival following taxane-anthracycline chemotherapy for invasive breast cancer. JAMA 305: 1873-1881, 2011.
19. Horak CE, Pusztai L, Xing G, Trifan OC, Saura C, Tseng LM, Chan S, Welcher R and Liu D: Biomarker analysis of neoadjuvant doxorubicin/cyclophosphamide followed by ixabepilone or paclitaxel in early-stage breast cancer. Clin Cancer Res 19: 1587-1595, 2013.

20. Shoemaker RH: The NCI60 human tumour cell line anticancer drug screen. Nat Rev Cancer 6: 813-823, 2006.

21. Subramanian A, Tamayo P, Mootha VK, Mukherjee S, Ebert BL, Gillette MA, Paulovich A, Pomeroy SL, Golub TR, Lander ES and Mesirov JP: Gene set enrichment analysis: A knowledge-based approach for interpreting genome-wide expression profiles. Proc Natl Acad Sci USA 102: 15545-15550, 2005.

22. Deng M, Yu R, Wang S, Zhang Y, Li Z, Song H, Liu B, Xu L, Wang $\mathrm{X}$, Zhang Z, et al: Limb-bud and heart attenuates growth and invasion of human lung adenocarcinoma cells and predicts survival outcome. Cell Physiol Biochem 47: 223-234, 2018.

23. Rao DD, Vorhies JS, Senzer N and Nemunaitis J: siRNA vs. shRNA: Similarities and differences. Adv Drug Deliv Rev 61: 746-759, 2009.

24. Jasra S and Anampa J: Anthracycline use for early stage breast cancer in the modern era: A review. Curr Treat Options Oncol 19: $30,2018$.

25. Ueno NT and Mamounas EP: Neoadjuvant nab-paclitaxel in the treatment of breast cancer. Breast Cancer Res Treat 156: 427-440, 2016

26. Morin X, Jaouen F and Durbec P: Control of planar divisions by the G-protein regulator LGN maintains progenitors in the chick neuroepithelium. Nat Neurosci 10: 1440-1448, 2007.

27. Culurgioni S, Mari S, Bonetti P, Gallini S, Bonetto G, Brennich M, Round A, Nicassio F and Mapelli M: Insc:LGN tetramers promote asymmetric divisions of mammary stem cells. Nat Commun 9: 1025, 2018.

28. Liu X, Wang J and Sun G: Identification of key genes and pathways in renal cell carcinoma through expression profiling data. Kidney Blood Press Res 40: 288-297, 2015.

29. Nowakowska A and Tarasiuk J: Invasion and metastasis of tumour cells resistant to chemotherapy. Postepy Hig Med Dosw (Online) 71: 380-397, 2017.

30. Kaur R, Kaur G, Gill RK, Soni R and Bariwal J: Recent developments in tubulin polymerization inhibitors: An overview. Eur J Med Chem 87: 89-124, 2014.

31. Wiser O, Qian X, Ehlers M, Ja WW, Roberts RW, Reuveny E, Jan YN and Jan LY: Modulation of basal and receptor-induced GIRK potassium channel activity and neuronal excitability by the mammalian PINS homolog LGN. Neuron 50: 561-573, 2006.

32. Churchill CD, Klobukowski M and Tuszynski JA: Elucidating the mechanism of action of the clinically approved taxanes: A comprehensive comparison of local and allosteric effects. Chem Biol Drug Des 86: 1253-1266, 2015.

33. Needleman DJ, Ojeda-Lopez MA, Raviv U, Ewert K, Miller HP, Wilson L and Safinya CR: Radial compression of microtubules and the mechanism of action of taxol and associated proteins. Biophys J 89: 3410-3423, 2005.

(i) (9) This work is licensed under a Creative Commons Attribution-NonCommercial-NoDerivatives 4.0 International (CC BY-NC-ND 4.0) License. 